\title{
Influence of size distribution and field amplitude on specific loss power
}

\author{
M. Boskovic, ${ }^{1, a)}$ G. F. Goya, ${ }^{2}$ S. Vranjes-Djuric, ${ }^{1}$ N. Jovic, ${ }^{1}$ B. Jancar, ${ }^{3}$ and B. Antic ${ }^{1}$ \\ ${ }^{1}$ The "Vinca" Institute of Nuclear Sciences, University of Belgrade, P.O. Box 522, 11001 Belgrade, Serbia \\ ${ }^{2}$ Instituto de Nanociencia de Aragon (INA), University of Zaragoza, Mariano Esquillor s/n, 50018 Zaragoza, \\ Spain \\ ${ }^{3}$ Jozef Stefan Institute, Jamova 39, 1000 Ljubljana, Slovenia
}

(Received 7 October 2014; accepted 23 February 2015; published online 9 March 2015)

\begin{abstract}
Herein we present the results of specific loss power (SLP) analysis of polydisperse water based ferrofluids, $\mathrm{Fe}_{3} \mathrm{O}_{4} / \mathrm{PEG} 200$ and $\mathrm{Fe}_{3} \mathrm{O}_{4} / \mathrm{PEG} 6000$, with average $\mathrm{Fe}_{3} \mathrm{O}_{4}$ particle size of $9 \mathrm{~nm}$ and $11 \mathrm{~nm}$, respectively. Specific loss power was measured in alternating magnetic field of various amplitudes and at fixed frequency of $580.5 \mathrm{kHz}$. Maximum SLP values acquired were $195 \mathrm{~W} / \mathrm{g}$ for $\mathrm{Fe}_{3} \mathrm{O}_{4} / \mathrm{PEG} 200$ and $60 \mathrm{~W} / \mathrm{g}$ for $\mathrm{Fe}_{3} \mathrm{O}_{4} / \mathrm{PEG} 6000$ samples. The samples were labeled as superparamagnetic by magnetization measurements, but SLP field dependence showed deviation from the behavior predicted by the commonly employed linear response theory. The scope of this theory for both samples with wide particle size distribution is discussed. Deviation from the expected behavior is explained by referring to polydisperse nature of the samples and field dependent relaxation rates. (C) 2015 AIP Publishing LLC. [http://dx.doi.org/10.1063/1.4914074]
\end{abstract}

\section{INTRODUCTION}

Magnetic hyperthermia is a therapeutic technique based on a fact that magnetic nanoparticles can heat the surrounding when exposed to an alternating magnetic field (AMF). An increasing number of works is being reported on means to accomplish large values of the specific loss power (SLP) of single-domain magnetic nanoparticles (MNPs), which is the key property reflecting their heating efficiency. Concurrently, new mathematical models have been developed to explain the heating mechanism under the specific conditions met by magnetic colloids under AMFs. ${ }^{1-3}$ The SLP of magnetic nanoparticles is measured by calorimetric techniques and expressed in $\mathrm{W} / \mathrm{g}$. The magnetic nanoparticles used for magnetic fluid hyperthermia applications are usually in the superparamagnetic state at room temperature, as in the case of the widely used $\mathrm{Fe}_{3} \mathrm{O}_{4}$ MNPs, with the size of up to $\sim 50 \mathrm{~nm}^{4,5}$

For superparamagnetic particles, the interpretation of the heating mechanisms can be done through the linear response theory (LRT). The main assumption of the LRT is that the Langevin function remains linear for the maximum field used in experiment. For this condition to be satisfied, the thermal energy must dominate over Zeeman energy ${ }^{6}$

$$
\frac{\mu B_{\max }}{k_{B} T}<1
$$

Here, $B_{\max }$ represents the maximum magnetic field amplitude and $\mu$ is the magnetic moment of the superparamagnetic particle which for spherical particles can be written as $\mu=M_{s} V=\frac{1}{6} M_{s} d^{3} \pi$, where $M_{s}$ stands for saturation magnetization and $d$ for the diameter of the particle. In the AC magnetic field, the magnetization lags behind the external field, so that $M(H(t))$ describes a hysteretic trajectory, defining an

\footnotetext{
a) Author to whom correspondence should be addressed. Electronic mail: markob@vinca.rs
}

internal area proportional to the magnetic energy loss. For any given applied field, $\mu_{0} H$, and frequency $\mathrm{f}$, the SLP can be computed as ${ }^{6}$

$$
S L P=f \times \text { hysteresis area }=f \frac{\pi \mu_{0}^{2} H^{2} M_{s}^{2} V}{3 k_{B} T} \frac{2 \pi f \tau_{R}}{1+\left(2 \pi f \tau_{R}\right)^{2}} .
$$

In ferrofluid samples, two relaxation mechanisms coexist-Brownian rotation of particles due to the random mechanical forces acting on particle inside the fluid (represented by relaxation time $\tau_{B}$ ) and Neel relaxation of particle's magnetic moment due to its weak coupling to the crystal structure (represented by relaxation time $\tau_{N}$ ). Effective relaxation time $\tau_{R}$ is then defined as $\frac{1}{\tau_{R}}=\frac{1}{\tau_{N}}+\frac{1}{\tau_{B}}$. In this model, the SLP shows square dependence on the applied field. In the low frequency regime, $f \ll \tau_{R}^{-1}$, the SLP changes quadratically with frequency. In the high frequency limit, $f \gg \tau_{R}^{-1}$, the SLP flattens and saturates to the value $\frac{\mu_{0}^{2} M_{s}^{2} V}{6 k_{B} T \tau_{R}} H^{2}$. Optimum frequency of the applied field is $1 / 2 \pi \tau_{R}$, where the expression $\frac{2 \pi f \tau_{R}}{1+\left(2 \pi f \tau_{R}\right)^{2}}$ reaches maximum.

For larger single-domain particles, the relaxation time lengthens and eventually surpasses the period of the applied AMF. Such particles cannot be regarded as superparamagnetic anymore, but rather as blocked. Behavior of these particles in the AC field is usually explained within Stoner-Wohlfarth based theories. $^{7}$ When $T=0$ or when the frequency of the applied field is very high, and when all the particles are oriented along the external field, the SLP field dependence shows step-like behavior-zero SLP while $\mu_{0} H<\mu_{0} H_{K}$, where $\mu_{0} H_{K}=\frac{2 K}{M_{s}}$ represents the anisotropy field, and exhibiting an abrupt jump to $f \cdot 4 M_{s} V \mu_{0} H_{C}$ when $\mu_{0} H \geq \mu_{0} H_{K}$.

In the case of randomly oriented easy axes, cumulative effect of particles with various orientations lowers the coercive field to $0.48 \mu_{0} H_{K}$ and suppresses the remanence $\mathrm{M}_{\mathrm{R}}$ to half of the saturation value. The area of the hysteresis loop is 
then $2 M_{s} V \mu_{0} H_{C}=0.96 M_{s} V \mu_{0} H_{K} \cdot{ }^{8-10}$ Finally, the inclusion of thermal relaxations in the model leads to coercive field that is dependent on temperature and extrinsic parameters such as field sweeping rate $^{11}$ and field amplitude. ${ }^{2,10}$ Nevertheless, the main trait of hysteresis loop area and the SLP field dependence stays no matter how complicated model one employs - opening of the loop and abrupt rise of the SLP when external field reaches some critical value, and subsequent saturation at higher fields. ${ }^{12,13}$

This paper presents the analysis of the influence of size distribution and applied field amplitude on the SLP. Mathematical model of heating power will be used to explain the calorimetrically measured data.

\section{EXPERIMENT}

\section{A. Sample preparation}

PEGylated $\mathrm{Fe}_{3} \mathrm{O}_{4}$ nanoparticles were prepared via modified Massart's method ${ }^{14}$ by chemical co-precipitation of iron(II) and iron(III) with $25 \% \mathrm{NH}_{4} \mathrm{OH}$. Polyethylene glycol (PEG, $\mathrm{Mw}=200$ and 6000) was first added to the initial solution of $0.1 \mathrm{M} \mathrm{FeCl}_{2} \cdot 4 \mathrm{H}_{2} \mathrm{O}$ and $0.2 \mathrm{M} \mathrm{FeCl}_{3} \cdot 6 \mathrm{H}_{2} \mathrm{O}$ (weight ratios 1: 1= PEG: iron oxide). Then, an ammonia aqueous solution was added dropwise and the suspension was heated up to $50^{\circ} \mathrm{C}$ under vigorous stirring. The coprecipitate was magnetically separated, washed several times with deionized water and finally re-dispersed in water. Prepared samples were accordingly labeled as $\mathrm{Fe}_{3} \mathrm{O}_{4} / \mathrm{PEG} 200$ and $\mathrm{Fe}_{3} \mathrm{O}_{4} /$ PEG6000.

\section{B. Experimental methods}

Fourier transform infrared spectroscopy (FTIR) was carried out at room temperature using a Nicolet 380 spectrophotometer operated in the spectral range of $4000-400 \mathrm{~cm}^{-1}$ with $4 \mathrm{~cm}^{-1}$ resolution. Further, the samples were characterized by thermogravimetric analysis (TGA) in order to determine the percentage of $\mathrm{Fe}_{3} \mathrm{O}_{4}$ in the dried samples. The TGA analyses were performed $\left(30-600^{\circ} \mathrm{C}\right.$ range) on a SDT Q600 TGA/DSC instrument (TA Instruments). The heating rate was $20^{\circ} \mathrm{C} \mathrm{min}^{-1}$ and the sample mass was less than $10 \mathrm{mg}$.

$\mathrm{X}$-ray powder diffraction (XRPD) data were collected on a Philips PW1710 diffractometer, in the angular range of $10-50^{\circ}(2 \theta)$ with a step size of $0.06^{\circ}$ and a counting time of $50 \mathrm{~s}$ per step. Transmission electron microscopy (TEM) measurements were carried out using a Jeol JEM 2100 HR electron microscope operating at $200 \mathrm{kV}$. The samples were prepared by ultra-sonication in ethanol and deposited on a conventional carbon-covered copper TEM grid. After drying, the samples were examined by TEM. Magnetic measurements of powder samples were performed on MPMS XL-5 SQUID magnetometer. Magnetization vs. temperature, $M$ (T), was measured in $2-300 \mathrm{~K}$ temperature range, under zero-field-cooled (ZFC) and field-cooled (FC) regimes, in $0.01 \mathrm{~T}$. Hysteresis loops were measured at $5 \mathrm{~K}$ and $300 \mathrm{~K}$ in ZFC regime.

Commercial AC applicator (model DM100 by nBnanoscale Biomagnetics) was used to measure the amount of magnetic field energy converted into heat. The temperature of ferrofluid samples subjected to the AC magnetic field was monitored using a fiber optic temperature probe and the SLP was calculated using equation $S L P=\frac{m_{W} c_{W}+m_{N P} c_{N P}}{m_{N P}}\left(\frac{\Delta T}{\Delta t}\right)$. Here, $m_{W}$ and $m_{N P}$ stand for mass of water and nanoparticles, where $c_{W}$ and $c_{N P}$ are their respective heat capacities. The mass of nanoparticles per volume of the sample was determined using spectrophotometric method. The ratio $\frac{\Delta T}{\Delta t}$ represents the initial rise of the temperature with time. Typically, data collected during the first $10-20 \mathrm{~s}$ of measurement, while the system can still be considered as adiabatic, were used and fitted by an exponential function. Measurements were repeated at least three times for each $H_{0}$ value chosen from the interval 50-300 G $(5-30 \mathrm{mT})$. The average SLP values (with the experimental errors) were plotted as a function of field amplitude at the frequency of $580.5 \mathrm{kHz}$.

\section{RESULTS AND DISCUSSION}

\section{A. Sample characterization}

Prepared PEGylated $\mathrm{Fe}_{3} \mathrm{O}_{4}$ samples formed stable water suspensions. The characteristics of the colloidal suspensions and involved nanoparticles were examined by different methods.

The mass of the adsorbed PEG layer on the surface of $\mathrm{Fe}_{3} \mathrm{O}_{4}$ nanoparticles was determined by TGA. The mass residues at $600{ }^{\circ} \mathrm{C}$ were found to be $95.1 \%$ and $94.5 \%$ for $\mathrm{Fe}_{3} \mathrm{O}_{4} / \mathrm{PEG} 200$ and $\mathrm{Fe}_{3} \mathrm{O}_{4} / \mathrm{PEG} 6000$, respectively (Figure 1). The presence of a PEG200/PEG6000 layer on magnetite surface was characterized by FTIR spectroscopy. FTIR spectra of $\mathrm{Fe}_{3} \mathrm{O}_{4} / \mathrm{PEG} 200$ and $\mathrm{Fe}_{3} \mathrm{O}_{4} / \mathrm{PEG} 6000$ are demonstrated in Figure 2 (black and green lines, respectively), while the FTIR spectrum of unmodified nanoparticles is shown by red line for comparison. The strong absorption peaks at $520 \mathrm{~cm}^{-1}\left(\mathrm{Fe}_{3} \mathrm{O}_{4} / \mathrm{PEG} 200\right)$ and $587 \mathrm{~cm}^{-1}\left(\mathrm{Fe}_{3} \mathrm{O}_{4} / \mathrm{PEG} 6000\right)$ are the characteristic absorption of $\mathrm{Fe}-\mathrm{O}$ bond, which confirmed the presence of magnetite. The distinct band at $840 \mathrm{~cm}^{-1}$ can be assigned to the in-plane $\mathrm{Fe}-\mathrm{O}-\mathrm{H}$ bending vibrations. ${ }^{15}$ For both coated $\mathrm{Fe}_{3} \mathrm{O}_{4}$ nanoparticles the bands around $2880 \mathrm{~cm}^{-1}$ (C-H asymmetric stretching), the peaks around $1620 \mathrm{~cm}^{-1}$ attributed to the carboxylate $(\mathrm{C}=\mathrm{O})$

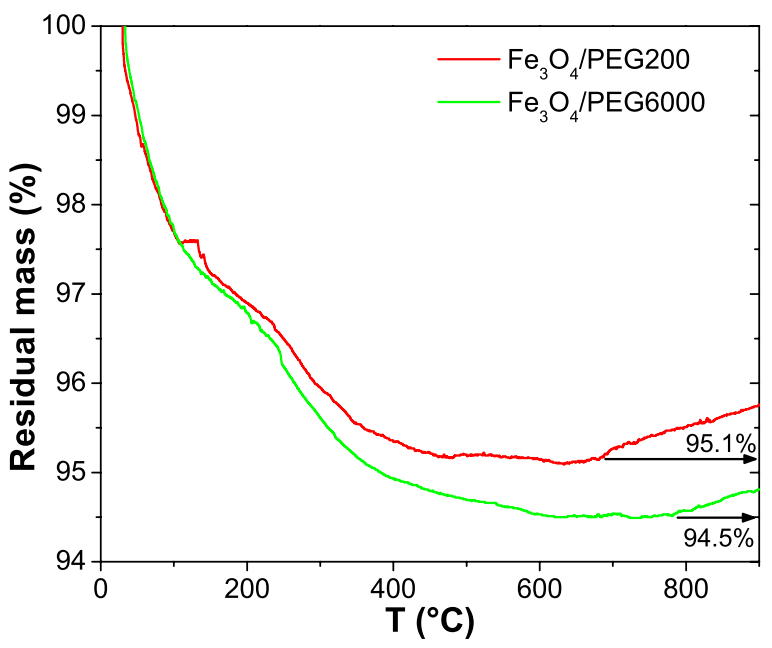

FIG. 1. TGA curves for Fe3O4/PEG200 and Fe3O4/PEG6000. 


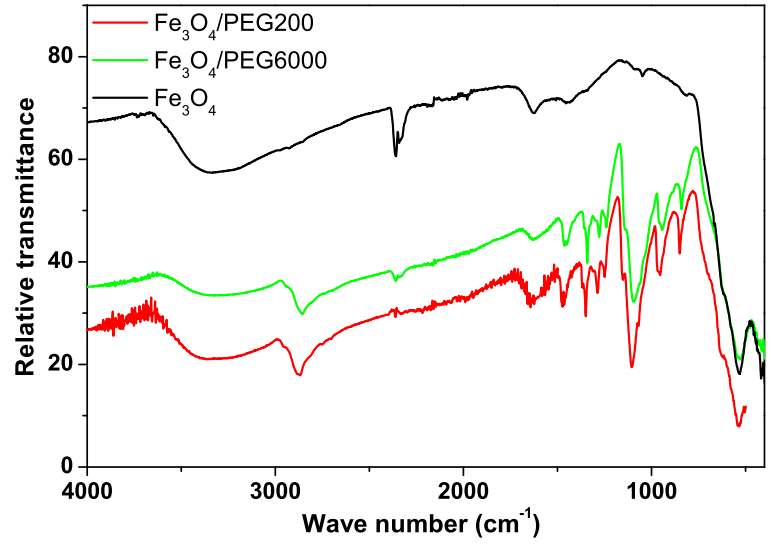

FIG. 2. FTIR spectra of PEGylated $\mathrm{Fe}_{3} \mathrm{O}_{4}$ samples and uncoated $\mathrm{Fe}_{3} \mathrm{O}_{4}$.

stretching vibrations, and the peaks at $1103 \mathrm{~cm}^{-1}$, which correspond to $\mathrm{C}-\mathrm{O}-\mathrm{C}$ stretching are the strong evidence for the presence of PEG molecules on the $\mathrm{Fe}_{3} \mathrm{O}_{4}$ surface. ${ }^{16}$

The X-ray diffraction patterns of PEGylated $\mathrm{Fe}_{3} \mathrm{O}_{4}$ nanoparticles are shown in the inset of Figure 3. The reflections are indexed within the expected spinel type structure of the space group $F d 3 m$. No additional reflections that would indicate potential presence of any other phase were observed, pointing to the single-phase nature of the samples. It is clear that the PEG, as coating material in both samples, did not affect crystalline structure of $\mathrm{Fe}_{3} \mathrm{O}_{4}$. The broad reflections indicate that the prepared samples are composed of ensembles of small size crystallites. We used Scherrer's formula to

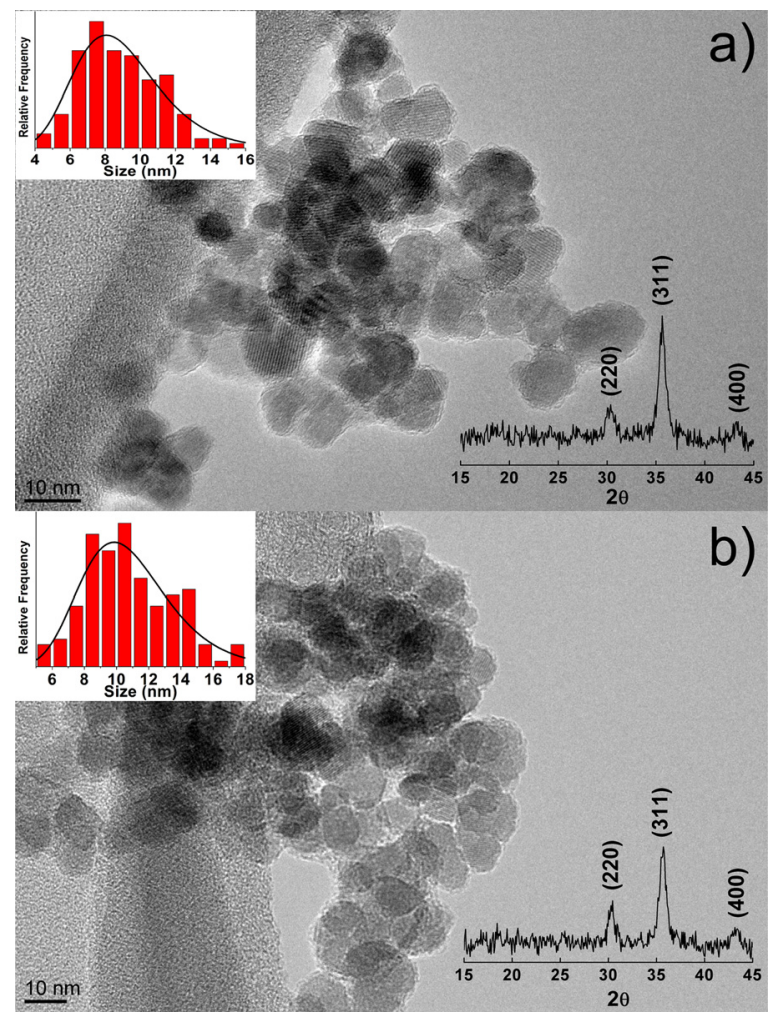

FIG. 3. TEM images (main panel) with number weighted size distribution histograms of PEGylated $\mathrm{Fe}_{3} \mathrm{O}_{4}$ samples (upper inset): (a) $\mathrm{Fe}_{3} \mathrm{O}_{4} / \mathrm{PEG} 200$ and (b) $\mathrm{Fe}_{3} \mathrm{O}_{4} / \mathrm{PEG} 6000$. Bottom inset: X-ray diffraction patterns. estimate crystallite size from the most intense (311) reflections: $\langle d\rangle=k \lambda /(\beta \cos \theta)$, where the shape factor $k$ is $0.9, \lambda$ is wavelength of $1.5418 \AA$ and $\beta$ is the full width at half maximum. The estimated crystallite size for $\mathrm{Fe}_{3} \mathrm{O}_{4} / \mathrm{PEG} 200$ is $10 \mathrm{~nm}$ and for $\mathrm{Fe}_{3} \mathrm{O}_{4} / \mathrm{PEG} 6000$ is $13 \mathrm{~nm}$.

TEM images and results of their analysis are shown in Figure 3. Images were analyzed in order to determine particle size, morphology, size distribution, and presence of PEG coating. The insets of Figure 3 show particle size distributions fitted by a lognormal function

$$
g_{n}(\bar{d}, \sigma, D)=\frac{1}{\sqrt{2 \pi} \sigma \frac{\mathrm{D}}{d}} \exp \left[-\frac{\ln \left[\frac{\mathrm{D}}{d}\right]^{2}}{2 \sigma^{2}}\right],
$$

where $D$ is particle diameter, and $\bar{d}$ and $\sigma$ are parameters of the distribution function.

The expected value and standard deviation for the diameter of $\mathrm{Fe}_{3} \mathrm{O}_{4} / \mathrm{PEG} 200$ and $\mathrm{Fe}_{3} \mathrm{O}_{4} / \mathrm{PEG} 6000$ samples are 9(2) $\mathrm{nm}$ and $11(3) \mathrm{nm}$, respectively. These results are close to the values obtained from Scherrer's formula suggesting that the majority of particles are composed from one crystallite. Concerning morphology, magnetite particles are observed to be approximately spherical in shape. PEG coating of the particles is visible as an amorphous shell around the crystalline particles (see Fig. 3).

\section{B. DC magnetization measurements}

The results of the DC magnetic measurements are shown in Figure 4. The hysteresis loops at $300 \mathrm{~K}$ of both samples are similar and typical for superparamagnetic systems, as can be seen in the main panel of the figure. The inset of Figure 4 shows coercivity fields $\left(H_{C}\right)$ at $5 \mathrm{~K}$. Coercivity field at $300 \mathrm{~K}$ is close to zero (below $0.3 \mathrm{mT}$ ) and increases as temperature drops. Saturation magnetization at $300 \mathrm{~K}$, calculated from $M\left(\frac{1}{H}\right)$ in the $\frac{1}{H} \rightarrow 0$ limit, is in the range of $72-74 \mathrm{Am}^{2} / \mathrm{kg}$ for both samples, which is close to the values usually reported for $\mathrm{Fe}_{3} \mathrm{O}_{4}$ MNPs. The zero-field-cooled

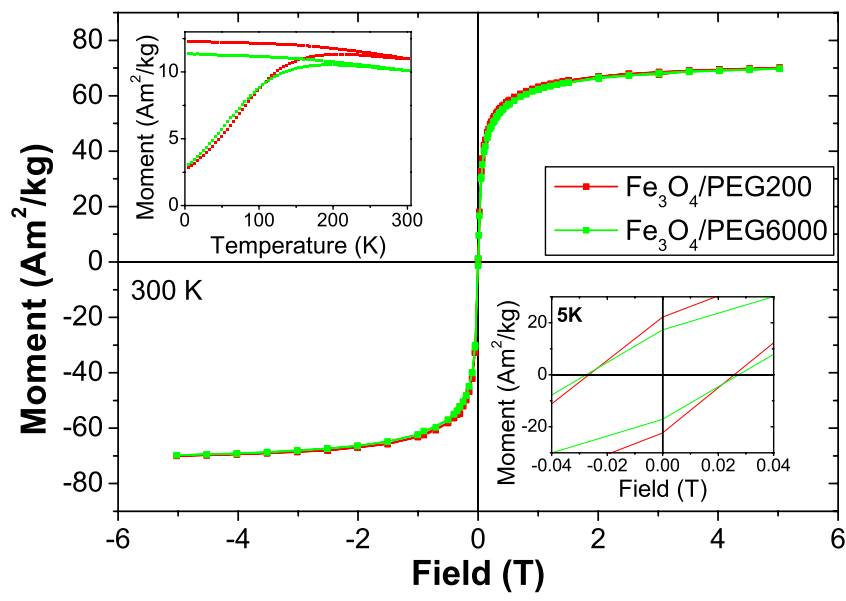

FIG. 4. Magnetic hysteresis of PEGylated samples at $300 \mathrm{~K}$. Inset in the upper left corner: temperature dependence of magnetization in $0.01 \mathrm{~T}$; inset in the lower right corner: part of the hysteresis loop at $5 \mathrm{~K}$. 
magnetization has a maximum in the range of 190-205 K (Figure 4, inset), while the temperature of irreversibility is close to $300 \mathrm{~K}$. This indicates that blocked particles exist even at room temperature.

In order to estimate the anisotropy field $\mu_{0} H_{K}$, temperature dependencies of coercive field $H_{C}$ were measured on $\mathrm{Fe}_{3} \mathrm{O}_{4} / \mathrm{PEG} 200$ and $\mathrm{Fe}_{3} \mathrm{O}_{4} / \mathrm{PEG} 6000$ samples. Figure 5 shows data and best fits using

$$
\mu_{0} H_{C}(T)=\mu_{0} H_{C}^{T=0}\left(1-\left(\frac{T}{\left\langle T_{B}\right\rangle}\right)^{1 / 2}\right),
$$

where $\left\langle T_{B}\right\rangle$ represents the average blocking temperature. ${ }^{17}$ We obtained values $0.07 \mathrm{~T}$ and $0.054 \mathrm{~T}$ for anisotropy fields, and from equation $\mu_{0} H_{K}=2 K / M_{S}$, the anisotropy constants are $14.5 \mathrm{~kJ} / \mathrm{m}^{3}$ and $11.4 \mathrm{~kJ} / \mathrm{m}^{3}$, for the two samples respectively. These results are comparable with values reported in the literature for nanosized magnetite. ${ }^{4,18,19}$ The decrease of anisotropy constant coincides with the increase in the average particle size in the studied samples. Additionally, coprecipitation is a low temperature synthesis, which can lead to incomplete crystallization of particles and to lower value of magnetocrystalline anisotropy.

\section{Size distribution, relaxation rates, and model validity}

Size distribution acquired from TEM analysis, though commonly used, is of limited value in calculations because properties and effects we investigate are generally volume dependent. This is why we should not be interested in how many particles of some diameter we have, but how large is their share in the total volume. Transition from TEM derived number weighted distribution $g_{n}(\bar{d}, \sigma)$ to volume weighted distribution is simple if we can approximate particles with some geometric bodies, e.g., spheres ${ }^{13}$

$$
g(\bar{d}, \sigma, D)=\frac{g_{n}(\bar{d}, \sigma, D) D^{3}}{\int g_{n}(\bar{d}, \sigma, D) D^{3} d D} .
$$

Volume weighted distribution is shifted towards larger values of diameter, and expected value is bigger than in the

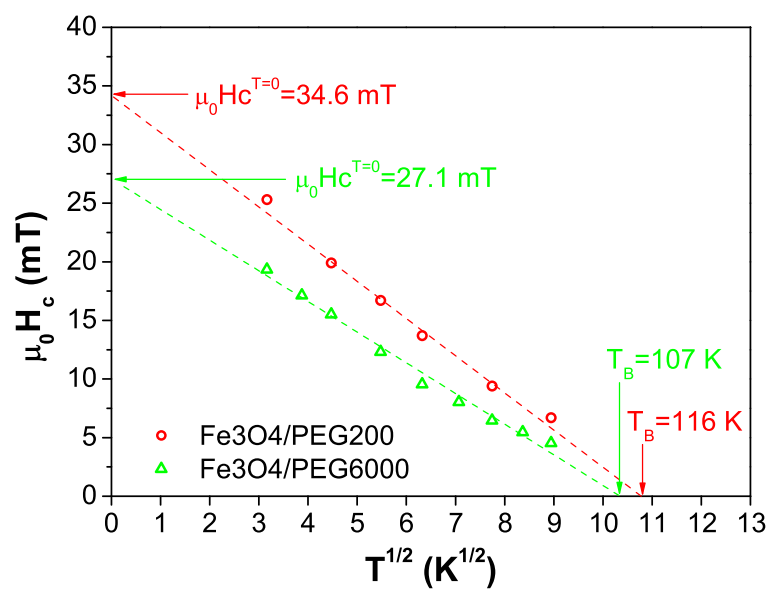

FIG. 5. $\boldsymbol{\mu}_{0} \boldsymbol{H}_{C}(\boldsymbol{T})$ dependence: symbols represent experimental data and lines represent best fits using Eq. (3). case of TEM derived distribution. In case of our samples, we get values of 11(3) nm for $\mathrm{Fe}_{3} \mathrm{O}_{4} / \mathrm{PEG} 200$ and 14(4) nm for $\mathrm{Fe}_{3} \mathrm{O}_{4} / \mathrm{PEG} 6000$. In subsequent calculations, where needed, volume weighted distributions are used.

Commonly used equations for calculation of relaxation times are $\tau_{N}=\tau_{0} \exp \left[\frac{K V}{k_{B} T}\right]$, for Néel relaxations and $\tau_{B}=\frac{3 \eta V_{H}}{k_{B} T}$, for Brown relaxations. Here, $\tau_{0}$ is of the order $10^{-10}$ to $10^{-9} \mathrm{~s}, \eta$ is viscosity of the carrier fluid, e.g., water, and $V_{H}$ is hydrodynamic volume of the particle. Mamiya and Jeyadevan ${ }^{4}$ suggested that relaxation times depend on amplitude of the external magnetic field and offered corrected equations

$$
\begin{aligned}
\frac{1}{\tau_{N}(H)}= & \frac{1}{\tau_{0}}\left(1-h^{2}\right)\left\{(1+h) \exp \left[\left(1+h^{2}\right)\left(\frac{-K V}{k_{B} T}\right)\right]\right. \\
& \left.+(1-h) \exp \left[\left(1-h^{2}\right)\left(\frac{-K V}{k_{B} T}\right)\right]\right\}
\end{aligned}
$$

and

$$
\frac{1}{\tau_{B}(H)}=\tau_{B}^{-1}\left[1+0.07\left(\frac{\mu_{0} M_{S} V H}{k_{B} T}\right)^{2}\right]^{1 / 2},
$$

where $h=\frac{H}{H_{K}}=\frac{H M_{S}}{2 K}$. Combined relaxation rate is $\frac{1}{\tau_{R}(H)}=\frac{1}{\tau_{N}(H)}+\frac{1}{\tau_{B}(H)}$. Because of the volume dependence, in size dispersed systems for each applied field there is a distribution of relaxation times. The size dependence of relaxation times for the highest and the lowest field used in our experiments is shown in Figure 6. The faster relaxation mechanism will dominate in the expression for combined relaxation time. Smaller particles typically relax via Néel, while larger relax via Brown mechanism. Size regions where Néel or Brown relaxations dominate can easily be seen. External field quickens the relaxation rates and causes the spreading of the Néel dominated region to larger particle sizes.

Tightly related to size dispersion is a question of validity of the employed models. In the case of LRT based model,

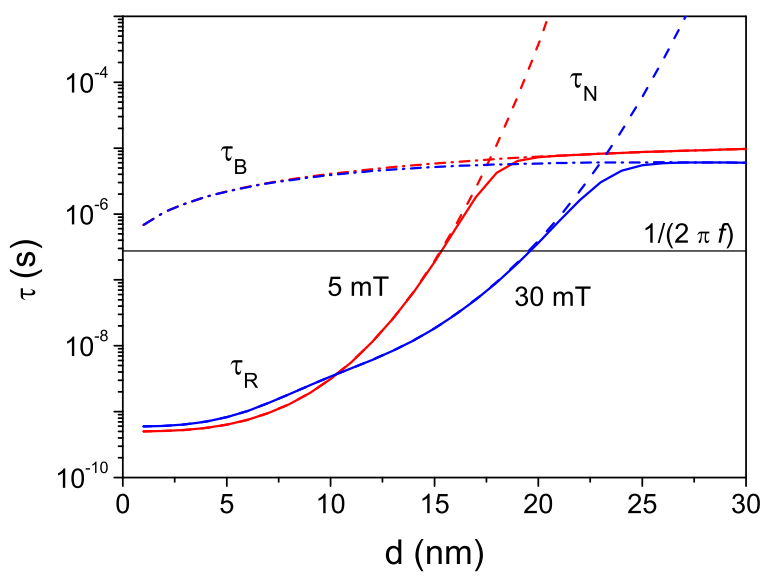

FIG. 6. $\mathrm{Fe}_{3} \mathrm{O}_{4} / \mathrm{PEG} 200$ sample relaxation times. Relaxation times are presented for the case of minimal field used in our experiments $(5 \mathrm{mT})$, depicted by red lines (left branch), and for the case of the maximal field $(30 \mathrm{mT})$, depicted by blue lines (right branch). Dashed lines correspond to Néel relaxation time $\left(\tau_{N}\right)$, dashed-dotted to Brown $\left(\tau_{B}\right)$, and full lines to combined relaxation time $\left(\tau_{R}\right)$. The straight horizontal line represents the period of external field, $\tau=1 /(2 \pi f)$. 


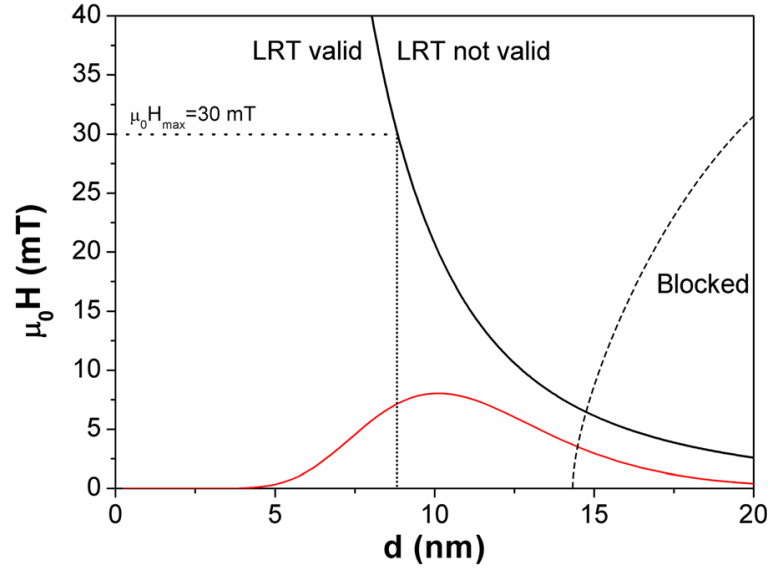

FIG. 7. LRT validity conditions for $\mathrm{Fe}_{3} \mathrm{O}_{4} / \mathrm{PEG} 200$ sample. Full black line represents $\mu(d) B=k_{B} T$ curve which delimits the area where LRT is applicable (left) and is not applicable (right); red bell-shaped line-volume weighted size distribution; dotted horizontal line-maximum field amplitude used in our experiments; and dashed line-relaxation condition $\tau_{R}=1 /(2 \pi f)$.

the linearity condition (Eq. (1)) gives connection between applied field amplitude and maximum particle size up to which the model can explain the behavior of the system. If depicted in the $d-B$ space (Figure 7) the equation $\mu(d) B=$ $k_{B} T$ defines a curve which delimits the area (under the curve) where the LRT is valid. ${ }^{20}$ Although the delimiting curve cannot be regarded as a sharp boundary, an important conclusion can be made. For every value of applied field, there is a maximum particle size for which the LRT can be employed. Due to the particle size distribution of our samples, as the field amplitude becomes larger the greater share of particles falls out of the area of validity of LRT and the experimental data generally should not follow the predictions of the model.

Another restriction comes from the fact that relaxation rate of particles in the system must be faster than the rate of change of the external field, $\tau_{R}(H)^{-1}>2 \pi f$ (Figure 7). Particles that do not fulfill this requirement must be regarded as blocked and their contribution to the heating has to be calculated outside the scope of LRT model.

\section{SLP measurements and discussion}

The SLP behavior of both samples was determined via calorimetric measurements in the AC magnetic field of various amplitudes and at fixed frequency of $580.5 \mathrm{kHz}$. The dependence of specific loss power on field amplitude is shown in Figure 8. The largest SLP values measured were $195 \mathrm{~W} / \mathrm{g}$ for $\mathrm{Fe}_{3} \mathrm{O}_{4} / \mathrm{PEG} 200$, and $60 \mathrm{~W} / \mathrm{g}$ for $\mathrm{Fe}_{3} \mathrm{O}_{4} / \mathrm{PEG} 6000$ sample. Up to approximately $0.01-0.015 \mathrm{~T}$ the curves follow the power law, then pass through inflection point and saturate at higher fields.

The acquired SLP data were fitted using equation

$$
S L P_{\text {fitt }}(H)=\int_{0}^{D_{f}(H)} g(\bar{d}, \sigma, D) \operatorname{SLP}(D, H) d D,
$$

where fitting parameters were $\bar{d}$ and $\sigma$. The $\operatorname{SLP}(D, H)$ function is given by Eq. (2), where we approximated nanoparticles with perfect spheres whose volume is given by

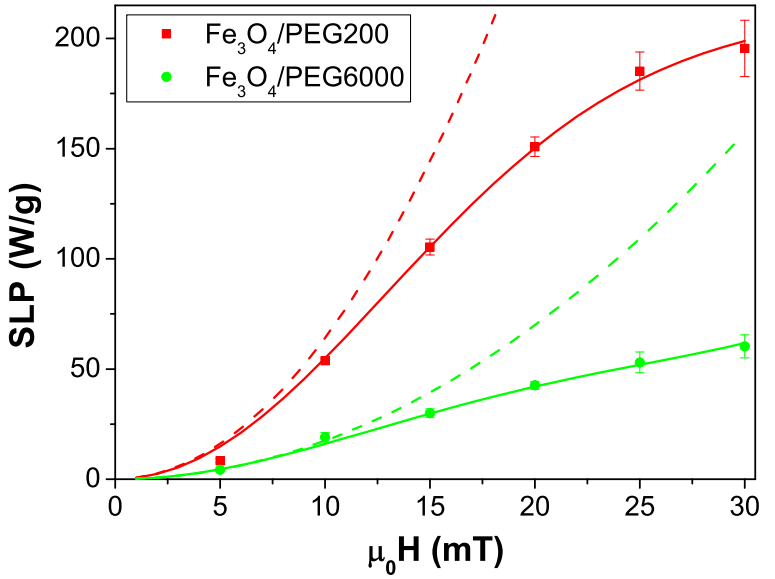

FIG. 8. Specific loss power for different amplitudes of applied alternating field. Symbols represent measured values, bars are experimental errors, full lines are best fits using size distribution functions and field dependent relaxation times. Dashed lines represent values of the SLP when field independent relaxation times are used.

$1 / 6 \pi D^{3}$. The initial values of parameters for volume weighted size distribution $g(\bar{d}, \sigma, D)$ were derived from TEM analysis results. Fitting curves are shown at Figure 8. The limit of integration, $D_{f}(H)$, is deduced from validity conditions. The linearity condition (Eq. (1)) has severe consequences, as it excludes large share of particles, depending on the applied field, and their significant contribution to heating is then unaccounted for (Figure 7). The omission of linearity condition in the calculation of SLP is a common practice, ${ }^{21,22}$ and the agreement of calculated data with measurements can be taken as a main justification for such action. Instead we used the weaker condition, $\tau_{R}(H)^{-1}>2 \pi f$ - only the particles relaxing faster than the rate of change of the external field are included in the calculations (see Figure 7). With this limit, integration covers $90 \%$ of particles at lowest used field, and more at higher fields. Contribution of blocked particles is expected to be largest at high fields, when external field approaches coercive field. But, due to the speeding of relaxations, share of blocked particles diminishes and their contribution can be omitted. Additionally, as can be seen from Figure 6, particles that satisfy this condition dominantly relax via Néel mechanism. This allows us to ignore the presence of a surface layer around particle, and to use volume of the particle instead of undetermined hydrodynamic volume in the calculation of Brown relaxation time.

Field dependent relaxation rates were used during the fitting procedure. For comparison, the SLP curves calculated with field independent relaxation times are presented in Figure 8. At higher fields, relaxation rates of particles become faster and relaxation frequency $1 / \tau_{R}$ is shifted further away from applied field frequency. This reduces the SLP and leads to saturation in the SLP field dependence.

Size distribution parameters, $\bar{d}$ and $\sigma$, were varied during the fitting procedure. The expected value and standard variation of the distribution for $\mathrm{Fe}_{3} \mathrm{O}_{4} / \mathrm{PEG} 200$ sample is 12 (2) $\mathrm{nm}$, and for $\mathrm{Fe}_{3} \mathrm{O}_{4} / \mathrm{PEG6000} \mathrm{10(3)} \mathrm{nm.} \mathrm{Result} \mathrm{for}$ $\mathrm{Fe}_{3} \mathrm{O}_{4} / \mathrm{PEG} 200$ agrees with the size determined from TEM analysis. On the other hand, the acquired average size of 
$\mathrm{Fe}_{3} \mathrm{O}_{4} / \mathrm{PEG} 6000$ is significantly smaller than the one derived from TEM. This points to the existence of magnetically disordered layer at the surfaces of the particles which do not contribute to the SLP. ${ }^{23,24}$ Due to a smaller magnetically active volume, larger size dispersion, lower anisotropy constant and heavy dependence of relaxation rates on these factors, $\mathrm{Fe}_{3} \mathrm{O}_{4} / \mathrm{PEG} 6000$ shows significantly lower SLP than $\mathrm{Fe}_{3} \mathrm{O}_{4} / \mathrm{PEG} 200$. The root of these differences between the samples is probably in synthesis conditions, since the coprecipitation technique, while simple and cheap, is hard to control and reproduce.

\section{CONCLUSION}

This article presents a quantitative analysis of specific loss power measurements with various amplitudes of applied alternating magnetic field. The analysis of the field dependent relaxation rates showed that the cause of saturation of the SLP at higher fields lies in the speeding of relaxations. Additionally, this speeding allowed us to neglect the contribution of blocked particles to the SLP and to use the LRT model for mathematical description of experimental data.

The results of the fitting procedure showed us how decisive the importance of size distribution and anisotropy constant of particles is. Relatively small variations in these factors can lead to a manifold change in heating power.

\section{ACKNOWLEDGMENTS}

Ministry of Education, Science and Technological Development of the Republic of Serbia supported this work financially through the Project's Grant No. III45015.
${ }^{1}$ R. Hergt, S. Dutz, R. Müller, and M. Zeisberger, J. Phys.: Condens. Matter 18, S2919 (2006).

${ }^{2}$ H. Mamiya, J. Nanomaterials 2013, 752973.

${ }^{3}$ A. E. Deatsch and B. A. Evans, J. Magn. Magn. Mater. 354, 163 (2014).

${ }^{4}$ H. Mamiya and B. Jeyadevan, Sci. Rep. 1, 157 (2011).

${ }^{5}$ D. J. Dunlop, J. Geophys. Res. 78, 1780, doi:10.1029/JB078i011p01780 (1973).

${ }^{6}$ R. E. Rosensweig, J. Magn. Magn. Mater. 252, 370 (2002).

${ }^{7}$ E. C. Stoner and E. P. Wohlfarth, Philos. Trans. R. Soc. A 240, 599 (1948).

${ }^{8}$ J. J. Lu, H. L. Huang, and I. Klik, J. Appl. Phys. 76, 1726 (1994).

${ }^{9}$ J. Carrey, B. Mehdaoui, and M. Respaud, J. Appl. Phys. 109, 083921 (2011).

${ }^{10}$ H. Pfeiffer, Phys. Status Solidi A 118, 295 (1990).

${ }^{11}$ N. A. Usov and Yu. B. Grebenshchikov, J. Appl. Phys. 106, 023917 (2009).

${ }^{12}$ B. Mehdaoui, A. Meffre, J. Carrey, S. Lachaize, L-M. Lacroix, M. Gougeon, B. Chaudret, and M. Respaud, Adv. Funct. Mater. 21, 4573 (2011).

${ }^{13}$ R. Hergt, S. Dutz, and M. Roder, J. Phys.: Condens. Matter 20, 385214 (2008).

${ }^{14}$ R. Massart, IEEE Trans. Magn. 17, 1247 (1981).

${ }^{15}$ J. Giri, T. Sriharsha, and D. Bahadur, J. Mater. Chem. 14, 875 (2004).

${ }^{16}$ A. Mukhopadhyay, N. Joshi, K. Chattopadhyay, and G. De, ACS Appl. Mater. Interfaces 4, 142 (2012).

${ }^{17}$ W. C. Nunes, W. S. D. Folly, J. P. Sinnecker, and M. A. Novak, Phys. Rev. B 70, 014419 (2004).

${ }^{18}$ R. M. Ferguson, K. R. Minard, A. P. Khandhar, and K. M. Krishnan, Med. Phys. 38, 1619 (2011)

${ }^{19}$ B. D. Cullity and C. D. Graham, Introduction to Magnetic Materials, 2nd ed. (Wiley, Hooben, 2009), p. 227.

${ }^{20}$ P. de la Presa, Y. Luengo, M. Multigner, R. Costo, M. P. Morales, G. Rivero, and A. Hernando, J. Phys. Chem. C116, 25602 (2012).

${ }^{21}$ E. Lima, Jr., E. De Biasi, M. V. Mansilla, M. E. Saleta, M. Granada, H. E. Troiani, F. B. Effenberger, L. M. Rossi, H. R. Rechenberg, and R. D. Zysler, J. Phys. D: Appl. Phys. 46, 045002 (2013).

${ }^{22}$ R. Muller, S. Dutz, A. Neeb, A. C. B. Cato, and M. Zeisberger, J. Magn. Magn. Mater. 328, 80 (2013).

${ }^{23}$ R. Kaiser and G. Miskolczy, J. Appl. Phys. 41, 1064 (1970).

${ }^{24}$ R. W. Chantrell, J. Popplewell, and S. W. Charles, IEEE Trans. Magn. 14, 975 (1978). 